\title{
A Role for Peroxidasin PXN-1 in Aspects of C. elegans Development
}

\author{
Juyeon Lee ${ }^{2,5}$, Jaya Bandyopadhyay, ${ }^{3,5}$, Jin II Lee ${ }^{4}$, Injeong Cho', Daeho Park', and Jeong Hoon Cho, *
}

\begin{abstract}
The Caenorhabditis elegans peroxidasins, $P X N-1$ and $P X N$ 2 , are extracellular peroxidases; pxn-2 is involved in muscleepidermal attachment during embryonic morphogenesis and in specific axon guidance. Here we investigate potential roles of the other homologue of peroxidasin, pxn-1, in C. elegans. A pxn-1 deletion mutant showed high lethality under heat-stress conditions. Using a transcriptional GFP reporter, pxn-1 expression was observed in various tissues including neurons, muscles, and hypodermis. A translational fusion showed that PXN-1::GFP was secreted and localized in extracellular matrix, particularly along body wall muscles and pharyngeal muscles. Various neuronal developmental defects were observed in pxn-1 mutants and in pxn-1 over-expressing animals, including handedness, branching, breakage, tangling, and defasciculation. These results suggest that pxn-1, like other peroxidasins, plays an important role throughout development.
\end{abstract}

\section{INTRODUCTION}

Peroxidasins are extracellular enzymes that contain a peroxidase domain and motifs of extracellular matrix (ECM) proteins, leucine-rich repeats (LRRs), and immunoglobulin (lg) domains. The peroxidasins' putative functions in host defense via antibacterial activity and in ECM formation have already been suggested and reviewed (Nelson et al., 1994; Soudi et al., 2012). Recent studies in the role that peroxidasins may play in tissue biogenesis have generated clinical and therapeutic interest as well (Peterfi and Geiszt, 2014). Despite their abundance among species, there have been few studies aimed at revealing peroxidasin's roles, especially in vivo (Nelson et al., 1994;

\footnotetext{
${ }^{1}$ Department of Biology Education, College of Education, Chosun University, Gwangju 501-759, Korea, ${ }^{2}$ Department of Life Science, Gwangju Institute of Science and Technology, Gwangju 500-712, Korea, ${ }^{3}$ Department of Biotechnology, West Bengal University of Technology, BF-142, Salt Lake City, Sector I, Kolkata 700-064, India, ${ }^{4}$ Division of Biological Science and Technology, College of Science and Technology, Yonsei University, Wonju 220-710, Korea, ${ }^{5}$ These authors contributed equally to this work.

${ }^{*}$ Correspondence: renocho@ chosun.ac.kr
}

Received 18 July, 2014; revised 21 October, 2014; accepted 24 October, 2014; published online 4 December, 2014

Keywords: C. elegans, heat stress, neuronal development, peroxidasins, pxn-1
Soudi et al., 2012).

There are two peroxidasin homologues, $\mathrm{PXN}-1$ and $\mathrm{PXN}-2$, in Caenorhabditis elegans (C. elegans), and in-depth research has revealed an important role for PXN-2 in embryonic morphogenesis, in muscle-epidermal attachment, and in adult axon regrowth following injury in worms (Gotenstein et al., 2010). Moreover, the peroxidase activity of PXN-2 has been shown to be critical for its function. A recent study has further shown that peroxidasins catalyze sulfilimine $(\mathrm{S}=\mathrm{N})$ bond formation between a methionine sulfur and a hydroxylysine nitrogen to strengthen the collagen IV network in the ECM (Bhave et al., 2012; Fidler et al., 2014; Gupta et al., 1997; Weiss, 2012). Therefore, peroxidasins in $C$. elegans are able to catalyze sulfilimine bond formation to strengthen epidermal-muscle attachments, and/or muscle-muscle attachments (Bhave et al., 2012).

To date, no specific roles in development have been ascribed to the pxn-1 gene and no function has been assigned to PXN-1 in $C$. elegans. It is thought that pxn-1 and pxn-2 may have an antagonistic functional relationship in $C$. elegans (Gotenstein et al., 2010), although conclusive evidence has not been presented yet. Furthermore, it is worth investigating the roles of $C$. elegans peroxidasins in detail, especially through characterization of pxn-1 mutants under stress conditions. Such conditions may be necessary to reveal a mutant phenotype not seen under normal conditions. Here we present an in-depth study on the role of pxn-1 in C. elegans through mutant and transgenic analysis in varying environmental conditions. Our results suggest that PXN-1 may have a role in the attachment of tissues and in neuronal guidance during development.

\section{MATERIALS AND METHODS}

\section{Strains and culture conditions}

The wild-type Bristol N2 strain, pxn-1(ok785) deletion mutant, and juls76(Punc-25::GFP) (Jin et al., 1999) were obtained from the Caenorhabditis elegans Genetics Center (CGC) at the University of Minnesota. Breeding of $C$. elegans was carried out according to published protocols (Brenner, 1974).

\section{Survival assay and heat stress}

For synchronization, gravid worms were treated with hypochlorite and then washed with M9 buffer several times. The eggs were used for the experiments. To measure survival rates, approximately 500 individual eggs of wild-type and pxn-1 (ok785) worms were grown to young adults at $20^{\circ} \mathrm{C}$ or $25^{\circ} \mathrm{C}$ (mild heat stress), and subsequently the live worms were counted. For severe $30^{\circ} \mathrm{C}$ heat stress (HS) experiments, wild- 

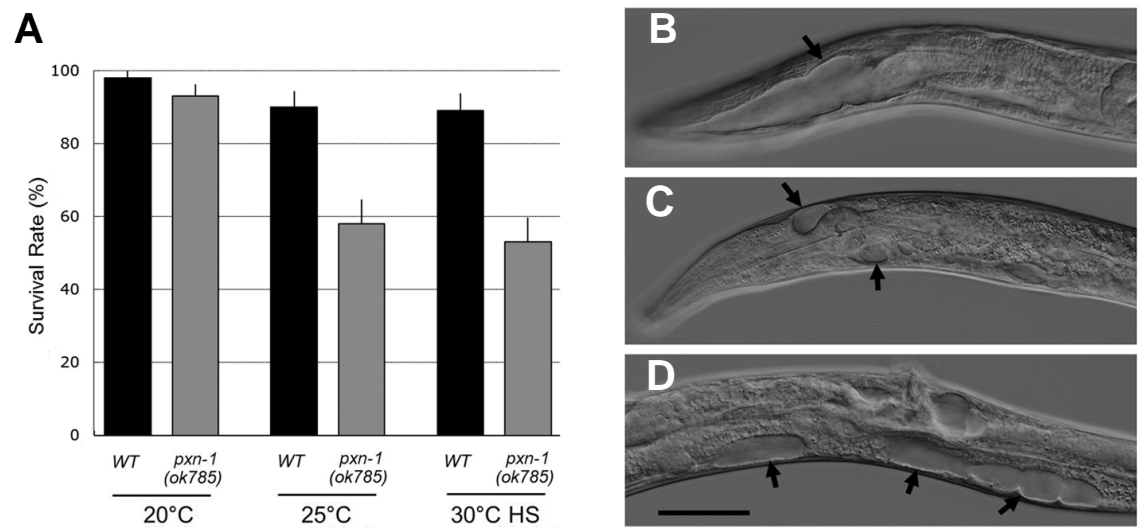

Fig. 1. Defective phenotypes of pxn-1 mutants under heat stress conditions. Wild-type (WT) and pxn-1(ok785) mutant were exposed constantly to normal or higher temperature at $20^{\circ} \mathrm{C}$ or $25^{\circ} \mathrm{C}$, respectively, except for heat stress at $30^{\circ} \mathrm{C}$. Heat treatment at $30^{\circ} \mathrm{C}$ was continued for $1 \mathrm{~h}$ in every $12 \mathrm{~h}$ throughout development from embryo to young adults. All young adults survived at $20^{\circ} \mathrm{C}, 25^{\circ} \mathrm{C}$ and $30^{\circ} \mathrm{C}$ were counted; mean \pm S.D. $n=500$ individuals per each line $(A)$. The severe phenotypes of pxn-1 mutant larvae under heat stress condition at $25^{\circ} \mathrm{C}$ are represented in head $(B, C)$ and mid-body (D). Arrows represent vacuoles. Scale bar, $50 \mu \mathrm{m}$. type and pxn-1(ok785) worms were incubated at $20^{\circ} \mathrm{C}$ as the baseline condition and were then subjected to heat stress at $30^{\circ} \mathrm{C}$ for $1 \mathrm{~h}$ every $12 \mathrm{~h}$.

\section{Constructs and transformation}

To prepare the transcriptional fusion constructs, approximately $3 \mathrm{~kb}$ of the 5 -upstream region of pxn-1 and $2.2 \mathrm{~kb}$ of pxn-2 were amplified by polymerase chain reaction and then inserted into a promoterless green fluorescent protein (GFP) vector pPD95.77, yielding Ppxn-1:: GFP and Ppxn-2:: GFP.

To prepare the translational fusion constructs, $p \times n-1$ and pxn-2 genomic DNA were ligated to $p x n-1$ and $p x n-2$ promoters with GFP and mCherry to yield Ppxn-1::pxn-1::GFP and Ppxn2::pxn-2::mCherry, respectively. For the quantification of axon abnormalities, Ppxn-1::pxn-2:::mCherry and Ppxn-2::pxn-1::GFP were constructed as well. The heat shock promoter from Fire vector (pPD49.83) was fused with the $p \times n-1$ and $p x n-2$ translational constructs, Phs::pxn-1::GFP and Phs::pxn-2::mCherry.

Microinjection was carried out as previously described (Mello and Fire, 1995). As a transformation marker, the plasmid pRF4 containing a dominant gene (rol-6) was co-injected with the translational fusion constructs into juls76(Punc-25::GFP). Transgenic worms were thereafter isolated by their rolling pattern and GFP expression was detected using a fluorescent microscope system (80i-DS-Fi1, Nikon).

\section{Assessing neuronal development}

The transgenic animals (at least 300 individuals per treatment) were cultured as described above. Worms overexpressing peroxidasins in response to heat shock (Phs::pxn-1::GFP and Phs::pxn-2:: mCherry) were subjected to heat shock at $30^{\circ} \mathrm{C}$ for $1.5 \mathrm{~h}$ every $12 \mathrm{~h}$. An individual worm that displayed at least one defect when observed under the microscope was scored as a neuronal defect. A defective neuron was defined as a neuron showing incorrect handedness, breakage, tangling, branching, or defasciculation.

To assess neuronal development in pxn-1 overexpressing strains (Phs::pxn-1::GFP) at different developmental stages, synchronized embryos, larvae, and adults were heat shocked once at $30^{\circ} \mathrm{C}$ for $1.5 \mathrm{~h}$ at different developmental stages. When they reached the young adult stage, the neuronal defects were scored. When the treatment was to be applied at the adult stage, worms were exposed to heat shock once they had reached the adult stage and their neuronal defects were counted one day later.

\section{RESULTS AND DISCUSSION}

Phenotypic analysis of C. elegans pxn-1 mutants Recently, Gotenstein et al. genetically characterized two $C$. elegans peroxidasins, $p \times n-1$ and $p x n-2$. In that study, various pxn-2 mutants were investigated in detail by characterizing eight mutants. These mutants had defects in epidermal elongation, muscle-epidermal attachment, and/or muscle-muscle attachment during development (Gotenstein et al., 2010). Although sequence alignment studies revealed a high homology between PXN-1 and PXN-2 proteins (52\% identity and $69 \%$ similarity, data not shown), their phenotypes were different, with pxn-2 mutants displaying obvious and severe defects and pxn1 mutants displaying no obvious phenotypes (Gotenstein et al., 2010). Such differences between the mutants suggest different roles for the two peroxidasins. Upon examination of the pxn-1 deletion mutant (ok785) for general characteristics such as growth rate (from egg to adult) and brood size, we found some differences between the pxn-1 mutant and wild-type $C$. elegans. At $20^{\circ} \mathrm{C}$, the pxn-1 mutant showed growth retardation compared to wild-type (N2), $70 \mathrm{~h}$ and $64 \mathrm{~h}$ at $20^{\circ} \mathrm{C}$ respectively. The brood size of the pxn-1 mutant was slightly smaller in comparison to wild-type, the two strains averaging 256 and 298, respectively. pxn-1 showed no obvious morphological defects under normal conditions, which is in agreement with the results from previous work (Gotenstein et al., 2010). However, when we varied conditions, we found some profound changes in the pxn-1 mutant. We examined pxn-1 phenotypes under stress conditions such as oxidative and heat stress. Treatment with the chemical paraquat, which can induce oxidative stress, did not cause any changes compared to wild-type (data not shown). However, high temperature dramatically increased lethality in the pxn-1 mutant. Wild-type and the pxn-1 mutant were grown at $20^{\circ} \mathrm{C}$ or $25^{\circ} \mathrm{C}$ (mild heat stress) from egg to adult. Worms grown under severe heat stress conditions were exposed to $30^{\circ} \mathrm{C}$ only for $1 \mathrm{~h}$ every $12 \mathrm{~h}$ from egg to adult because harsh temperatures normally cause death in $C$. elegans. Wild-type worms showed little difference in their survival rates, either at $25^{\circ} \mathrm{C}(90 \%)$ or at $30^{\circ} \mathrm{C}(89 \%)$ compared to survival at $20^{\circ} \mathrm{C}$ $(98 \%)$ (Fig. 1A). The mutant was more susceptible to heat stress-mediated lethality, however, exhibiting $58 \%$ survival at $25^{\circ} \mathrm{C}$ and $53 \%$ at $30^{\circ} \mathrm{C}$ but $93 \%$ survival at $20^{\circ} \mathrm{C}$.

We also observed that heat stress conditions caused the formation of large vacuoles in the pxn-1 mutant, particularly between the pharynx and the epidermis in the anterior region 

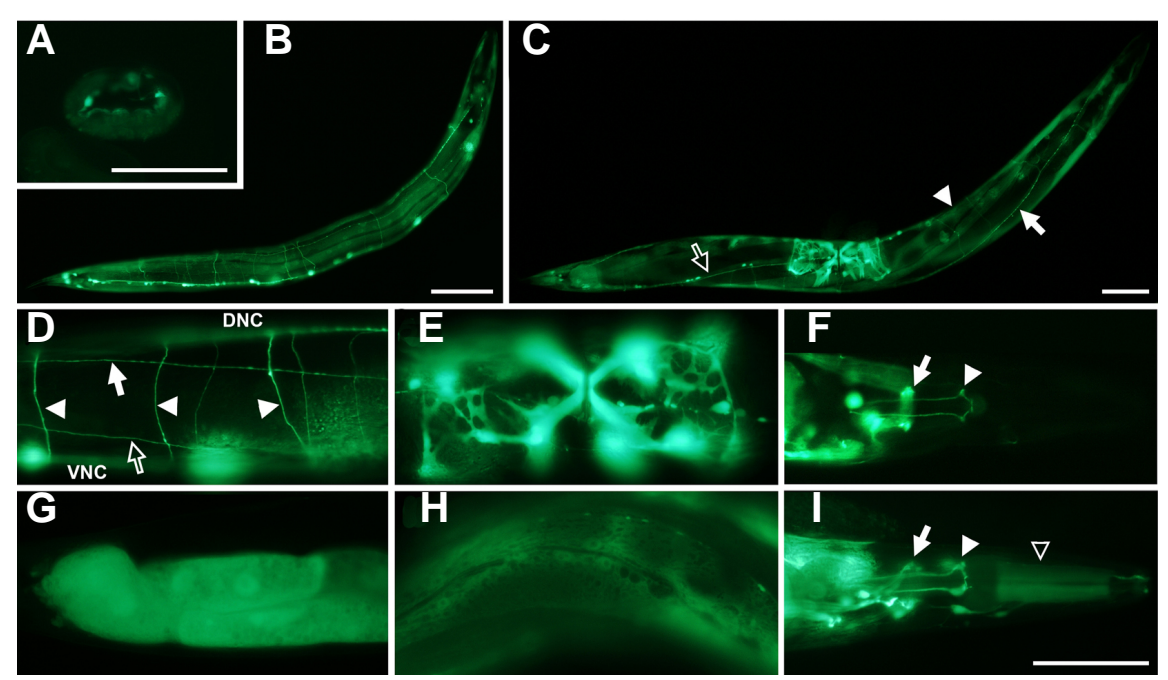

Fig. 2. Expression of pxn-1. Ppxn$1:: G F P$ transcriptional fusion constructs were injected into wild-type and pxn-1 mutants. In wild-type worms, pxn-1 expressed throughout development, from an embryo through adult stages [(A), embryo; (B), Larva L3; and C, adult; arrowhead, body wall muscle; open arrow, ventral nerve cord (VNC); solid arrow, dorsal nerve cord (DNC) in (C)] and in various tissues [(D), neurons; $(E)$, vulva and uterine muscles; $(F)$, head neurons; $(G)$, intestine and (H), hypodermis]. In (D), ALN, PLN, and GABAergic neurons were represented as an arrow, an open arrow and arrowheads respectively. In (F) and (I), arrows represent nerve ring and solid arrowheads pharyngeal neurons. In pxn-1 mutants (I), the expression was observed in head neurons and pharyngeal muscles [pm3, open arrowhead in (I)]. Scale bars, $50 \mu \mathrm{m}$ in (A-C), and (D-I).

(arrows in Figs. 1B and 1C) and between the gonad and the epidermis in the mid-body section (arrows in Fig. 1D). We observed this phenotype in $23.5 \%$ of pxn-1 mutant animals and in only $5.5 \%$ of the wild-type animals. Moreover, since the pxn-1 mutants had high lethality, and since we were only able to observe the surviving young adults, the prevalence of vacuole formation may actually be higher than the $23.5 \%$ we observed.

According to Gotenstein et al. (2010) pxn-2 adults showed a similar vacuole phenotype around the pharyngeal region under normal conditions. These vacuoles may be the result of weak attachments and/or cell necrosis between tissues due to breaching of the ECM. As the worm moves along using its body muscles, detachment of tissue becomes even more magnified. We conjectured that increased temperature had induced hyperactive movement in the worms, thereby magnifying detachment and/or necrosis phenotypes. To test whether this may also be the case for pxn-1 mutants, we paralyzed the worms by treatment with levamisole, an agonist of the acetylcholine receptor. However, neither wild-type nor pxn-1 animals had any apparent improvement in their survival rates or vacuole phenotypes upon levamisole treatment.

Since one of peroxidasin's proposed roles is in host defense via antibacterial activity, we tested whether the increased lethality is due to infection by $E$. coli. The OP50 strain of $E$. coli is normally used in the laboratory as food for bacteriovorous $C$. elegans; however, under some conditions OP50 can be toxic to the worms. We fed UV-killed OP50 to pxn-1 to test whether the increased lethality in pxn-1 results from weak host defense. No difference in survival rates was found between live and UV-killed bacteria feeding (data not shown). Our experiments here indicate that neither excessive movement nor bacterial infection is the direct cause of the observed lethality under stress conditions.

On the other hand, several studies demonstrate that disturbances in ECM structure can result in morphological and development phenotypes in C. elegans (Peters et al., 1991; Thacker et al., 1995; 2000; Topf and Chiquet-Ehrismann, 2011). From observations and available reports, a correlation can be drawn between the vacuole phenotypes and increased lethality, which indicates important roles of pxn-1 in ECM structural integrity and function under heat stress conditions. pxn-1 is expressed throughout developmental stages and in various tissues

To better understand the roles of pxn-1 in $C$. elegans, the pxn-1 gene expression pattern was observed using pxn-1 transcriptional GFP reporters (Ppxn-1::GFP) injected into either wildtype or pxn-1 mutants. Wild-type transgenic animals showed a constant expression of pxn-1 throughout their development, starting from the egg and continuing through to the adult stages (Figs. 2A-2C). The expression patterns were associated with the ventral nerve cord (VNC), the dorsal nerve cord (DNC), body wall muscles (open arrow, arrow, and arrowhead, respectively, in Fig. 2C), neurons, vulval muscles, uterine muscles, head neurons, intestine, and hypodermis (Figs. 2D-2H, respectively). These expression patterns were consistent with the Ppxn-1-GFP expression pattern seen in a previous study (Gotenstein et al., 2010). In D, lateral cholinergic neurons are visualized-ALN and PLN (arrow and open arrow)-as well as GABAergic neurons, including commissures (COs, arrowheads). Moreover, the patterns were observed in excretory cells and in the spermathecal-uterine valve (data not shown). An arrow and an arrowhead in Fig. $2 \mathrm{~F}$ indicate the nerve ring and a pharyngeal neuron, respectively. In the pxn-1 mutant, pharyngeal neurons displayed increased GFP expression levels (arrowhead, Fig. 2l). In addition, the pharyngeal muscle (pm3) became visible in the pxn-1 mutant (open arrowhead, Fig. 2l). These enhanced GFP expressions in mutant animals may result from upregulation of pxn-1 gene expression due to a lack of functional PXN-1 proteins. They could also indicate reciprocal regulation of $p x n-1$ by $p x n-2$ in light of the proposed antagonistic relationship between these two proteins (Gotenstein et al., 2010).

\section{$P X N-1:: G F P$ is localized in extracellular matrix (ECM)}

The previous study by Gotenstein et al. (2010) showed that PXN-2 is localized to the epidermis and plays an important role in muscle-epidermal attachment. We also observed possible attachment defects in pxn-1 mutants under heat stress conditions, indicating a need for careful examination of PXN-1 protein localization. We constructed a GFP-tagged transgene containing the pxn-1 promoter and pxn-1 gene (Ppxn-1::pxn$1:: G F P)$ or an mCherry-tagged pxn-2 promoter and the pxn-2 

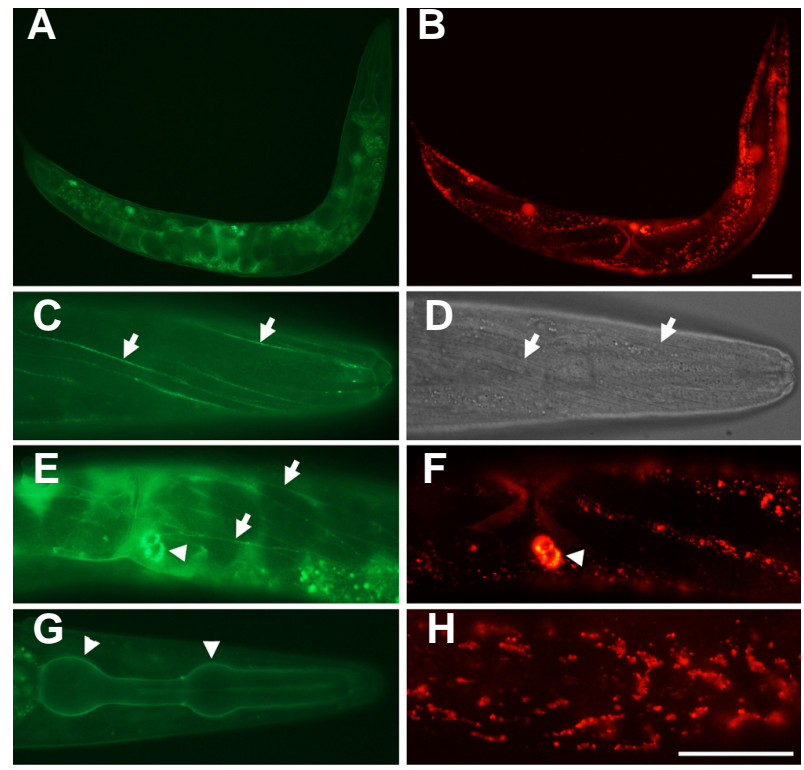

Fig.3. Localization of PXN-1 and PXN-2. Ppxn-1::pxn-1::GFP and Ppxn-2::pxn-2::mCherry translational fusion constructs were coinjected into wild-type. PXN-1 localization is represented by the GFP expressions [(A), (C), (E), and $(G)]$, and PXN-2 localization is represented by mCherry $[(B),(F)$, and $(H)]$. GFP expressions were intense in muscle-muscle attachment regions [arrows in (C), (D), and $(E)]$. The same image of $(C)$ was represented as a DIC image in $(D)$. Arrowheads in $(E)$ and $(F)$ represent coelomocytes. PXN-1 is localized along with pharynx [arrowhead in $(\mathrm{G})$ ], and PXN-2 in hypodermis $(H)$. Scale bar, $50 \mu \mathrm{m}$ in $(A, B)$ and $(C-H)$.

gene (Ppxn-2::pxn-2::mCherry). These constructs were coinjected into wild-type animals. PXN-1::GFP expressed all along the body of the animal (Fig. 3A), but the expression was more distinctly found in the muscle-muscle attachment regions (arrows in Figs. 3C and 3D; D, DIC image of C). Conversely, PXN-2::mCherry expressed in regions somewhat different from those that were found to express PXN-1 (Fig. 3B). Specifically, PXN-1::GFP expression localized between body wall muscle cells (arrows in Fig. 3E), but such expression was not observed for PXN-2::mCherry (Fig. 3F, the same image of E). PXN-1 and PXN-2 were localized in the coelomocytes (arrowheads in Figs. $3 \mathrm{E}$ and $3 \mathrm{~F}$ ) and PXN-2 was found in the hypodermis (Figs. 3F and $3 \mathrm{H}$ ). Interestingly, PXN-1::GFP was distributed along the pharynx (Fig. 3G), which signifies that PXN-1 may function in the attachments between pharynx and hypodermis. The localization of PXN-1 is consistent with the location of the vacuoles shown in Fig. 1C (arrows) along the pharynx. In addition, since PXN-1 and PXN-2 were both localized to the coelomocytes, it appears that both are likely secretory proteins (Figs. 3E and 3F).

\section{$P X N-1$ is involved in neuronal development}

We showed that pxn-1 is prominently expressed in neurons, including GABAergic and cholinergic neurons. We therefore asked whether pxn-1 has any roles in neuronal development. We used the pxn-1 promoter region (Ppxn-1::GFP) in wild-type, pxn-1 mutants, and pxn-1 over-expressing worms. In the wildtype transgenic animals, commissures (CO) at the anterior end of the body connect from the ventral nerve cord (VNC) to the dorsal nerve cord (DNC) and vice versa. In the same way, COs on the left side of the body (DD1 and VD2, arrowheads in Figs.
$4 \mathrm{~A}-4 \mathrm{C}$ ) are connected in a similar manner to the right side of the body (VD3 to VD13 and DD2 to DD6, arrows in Fig. 4B). On the other hand, pxn-1 mutants displayed wrong-sided handedness of the COs (see many arrows in Fig. 4C). Moreover, pxn1 overexpressing animals (Ppxn-1::pxn-1::GFP) showed a similar pattern, with many COs located on the wrong side (Fig. 4D). Some of them also showed over-branching at the ends of neurons (arrowheads in Fig. 4D, inset). High-magnification images illustrated prominent breakages in ALN (a cholinergic sensory neuron) in the pxn-1 mutant (arrows in Fig. 4F). Furthermore, the abnormal patterns of COs, for instance, tangled neurons (arrowheads in Fig. 4G), misguided neurons (arrows in Figs. $4 \mathrm{G}$ and $4 \mathrm{H}$ ), and disconnected neurons (open arrow in Fig. $4 \mathrm{H}$ ) observed in overexpressing animals (Ppxn-1::pxn-1, Figs. 4G and $4 \mathrm{H}$ ) were rarely observed in wild-type animals (Fig. 4E). To confirm the role of PXN-1 in neuronal development, we ectopically expressed pxn-1 using a heat shock promoter (Phs:::pxn$1:: G F P)$. Expression of this construct alone without heat shock showed no differences compared to wild-type. However, upon heat shock, defects in neuronal navigation were clearly apparent (Figs. 4J and 4K). In animals ectopically expressing pxn-1 by heat shock, the COs not only displayed loss of direction but were also shown to tangle with each other (arrowheads in Figs. $4 \mathrm{~J}$ and $4 \mathrm{~K}$ ). Abnormal arrangements such as defasciculated neurons were also found in the pxn-1 mutant (arrow in Fig. 4I) as well as in the animals ectopically expressing pxn-1 (arrow in Fig. 4J). Because juls76(Punc-25:: GFP) is expressed specifically in GABAergic neurons (Jin et al., 1999), we also used juls76 to confirm the neuronal developmental defects in the pxn-1 mutant and in over-expressing animals. Using the Punc25::GFP we were able to confirm all the neuronal defects we observed using Ppxn-1::GFP in the pxn-1 mutant and in overexpressing animals.

The abnormal neuronal development defects-meandering, breakage, branching, and defasciculated COs-have previously been observed in mutants characterized by unstable ECM structure (Knobel et al., 1999; Poinat et al., 2002). This is in agreement with the fact that axonal guidance is dependent upon various cues such as signaling molecules or structural molecules situated in the ECM (Dickson, 2002; Mueller, 1999; Tessier-Lavigne and Goodman, 1996). Hence, from these qualitative observations and existing reports, we think that PXN-1 plays important roles in neuronal development via an influence on ECM integrity.

We further quantified the neuronal development abnormalities using the juls76(Punc-25::GFP) strain. When compared with wild-type (juls76), the corresponding pxn-1 mutant (pxn-1 juls76) demonstrated increased neuronal defects (32\% and $48.7 \%$ of animals affected, respectively, Fig. 5A). Moreover, pxn-1 overexpressing animals under the control of a pxn-1 promoter (Ppxn-1::pxn-1::GFP) showed a drastic increase in axonal abnormalities to about $72.3 \%$. We also swapped promoters and overexpressed either pxn-1 with a pxn-2 promoter (Ppxn-2::pxn-1::GFP) or pxn-2 with a pxn-1 promoter (Ppxn$1::$ pxn-2::mCherry). Neither of these showed much difference from wild-type. These results indicate that peroxidasin's expression under the proper promoter and in the proper location is critical for its function.

Acute overexpression of pxn-1 using a heat-shock promoter (Phs::pxn-1::GFP) was also effective in increasing the prevalence of aberrations, by about $69 \%$ (Fig. 5B). Thus, increased neuronal defects in pxn-1 mutant and pxn-1 overexpressing animals (cis- or ectopic) indicate that $\mathrm{PXN}-1$ plays an important role in neuronal development. We also expressed pxn-2 under: 

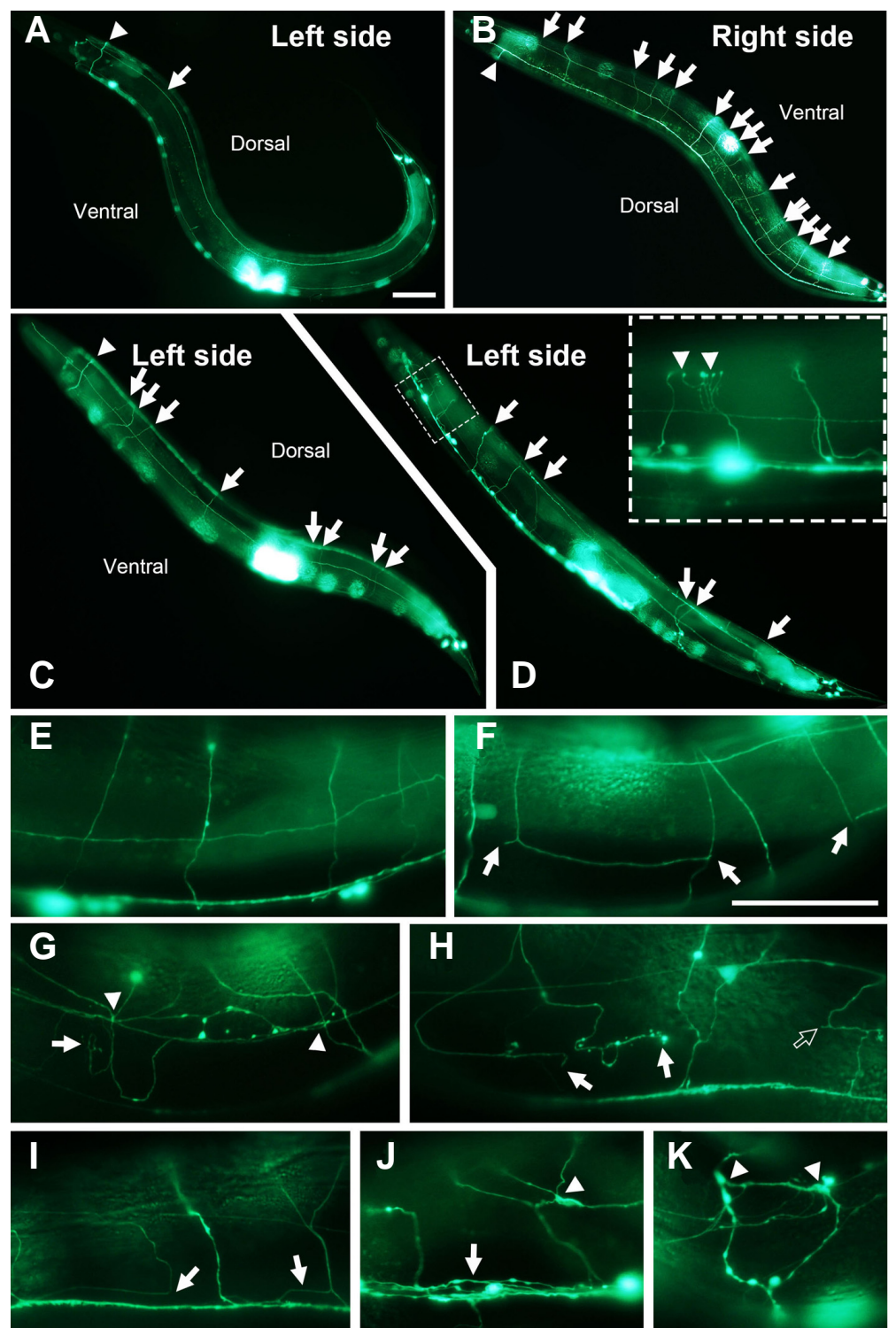

Fig. 4. Defects of axonal navigation in pxn-1 mutants and overexpressing animals. In wild-type transgenic animals, ALN, cholinergic neuron (arrow) and DD1 and VD2 neuron [arrow head, the same applied to $(B, C)$ ] were located on the left side $(A)$ and arrows represented GABAergic neurons (VD3VD13 and DD2 - DD6) on the right side (B). In pxn1 mutant and overexpressing worms (Ppxn-1::pxn$1:: G F P)$, motor neurons [arrows in $(C, D)$ respectively] were extended on the wrong side, the left. Inset of (D) was the enlarged image of the dotted box and the arrowheads showed over-branching of nerve ends. Representative neuron images of wildtype (E), pxn-1 mutant (F, I), Ppxn-1::pxn-1::GFP $(\mathrm{G}, \mathrm{H})$, and Phs::pxn-1::GFP (J, K): axonal breakage [arrows in $(F)$ and open arrow in $(H)$ ], prematurely branching [arrows in $(\mathrm{G})$ and $(\mathrm{H})$ ], tangling [arrowheads in (G, J, and $\mathrm{K})$ ], defasciculation [arrows in $(\mathrm{I}, \mathrm{J})$ ]. Scale bars, $50 \mu \mathrm{m}$ in A-D and E-K. the control of various promoters (Ppxn-1::pxn-2::mCherry, Ppxn2::pxn-2::mCherry and Phs:pxn-2::mCherry). As mentioned previously, there were no apparent effects in Ppxn-1::pxn2::mCherry neurons. However, pxn-2 overexpressing animals (either cis- or ectopic) had increased axonal defects. This suggests some relationship between PXN-1 and PXN-2 consistent with what has been previously proposed (Gotenstein et al., 2010).

In addition, we asked whether PXN-1's role in neuronal development is specific to a critical period in development, or whether it plays a general role. Accordingly, we used transgenic worms that expressed pxn-1 under a heat shock promoter (Phs::pxn-1::GFP) and applied heat shock at different developmental stages to assess the role of PXN-1 throughout development. Neuronal defects of pxn-1 ectopic expression were much more prominent at early developmental stages, i.e., from embryo to larval stages (Fig. 5C), which indicates that pxn-1 plays critical roles in neuronal development at early developmental stages. Moreover, we observed that pxn-1 was ex- pressed constantly throughout the adult worm's lifetime, (data not shown), which could indicate involvement in neuronal maintenance during the aging process. Thus, the role of PXN-1 in neuronal development is acute and may be stage-specific during development and throughout the lifetime of the worm.

Our previous analysis of the pxn-1 mutant indicated that it was sensitive to heat stress. Thus, we also scored neuronal defects in the pxn-1 mutant animals that survived at $25^{\circ} \mathrm{C}$ (Fig. $5 D$ ). Moreover, even in wild-type worms heat stress alone induced neuronal developmental defects (Fig 5D). In the pxn-1 mutant, heat stress did not cause any synergistic increases in neuronal defects compared to wild-type at $25^{\circ} \mathrm{C}$ (Fig 5D) or at $30^{\circ} \mathrm{C}$ (data not shown). However, once again, heat stress led to high lethality in the pxn-1 mutant such that the prevalence of neuronal defects may actually have been much higher than our results indicate.

Our results further indicate that pxn-1 function is different from that of $p \times n-2$, at least in the context of neuronal navigation. 
A

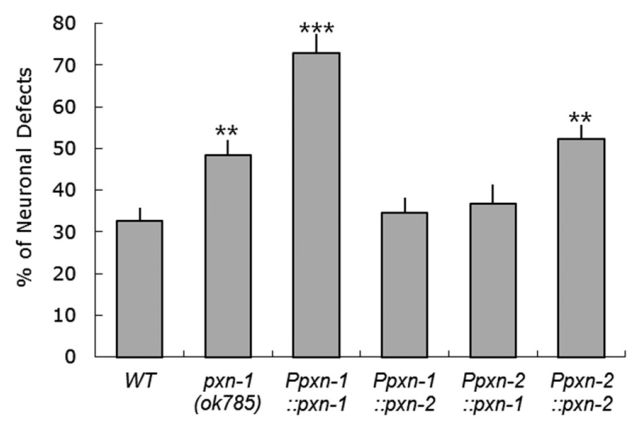

C

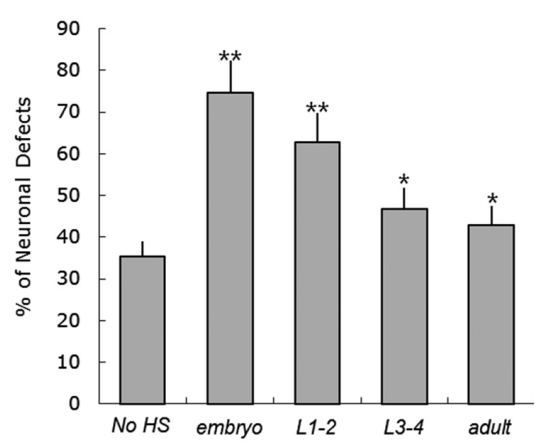

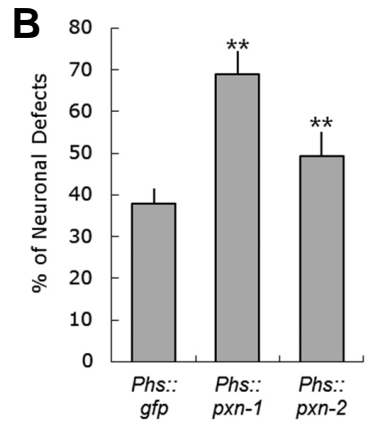

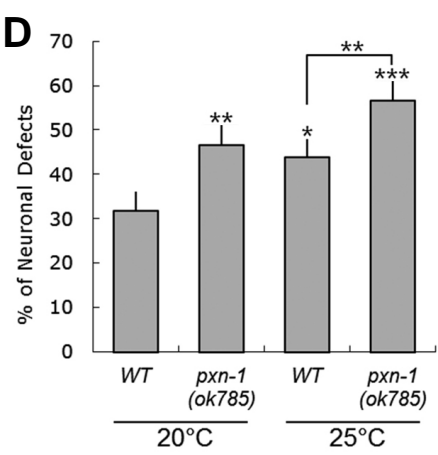

Fig. 5. The degree of incidence of neuronal defects in various transgenic animals, different developmental stages, and heat stress condition. Abnormal axonal occurrences in wild-type, pxn-1 mutants, Ppxn1::pxn-1, Ppxn-1::pxn-2::mCherry, Ppxn-2::pxn-1, and Ppxn-2::pxn-2::mChemy were represented as percentage (A). Neuronal defects in ectopic pxn-1::GFP and pxn-2::mCherry over-expression (Phs:: pxn-1::GFP and Phs::pxn-2::mCherry) were represented as percentage $(B)$. The percentage of abnormal neurons in pxn-1 ectopic expression (Phs::pxn-1::GFP) at different developmental stages were represented; egg, L1-L2, L3-L4, and adult $(C)$. Temperature effects on axon abnormality in wild-type and pxn-1 mutants (D). All experiments were performed in the juls76(Punc-25::GFP) line. $\mathrm{N}>300$ individuals per each line. Error bar = STD. ${ }^{\star} p<0.05 ;{ }^{* \star} p<0.01 ;{ }^{\star \star \star} p<$ 0.001 ; Student's $t$-test.
These results are consistent with the rescue experiments reported earlier, wherein a peroxidase domain of pxn-1 was unable to rescue pxn-2 mutant phenotypes (Gotenstein et al., 2010). An inability to rescue pxn-2 mutant phenotypes may be a consequence of antagonistic roles for pxn-1 and pxn-2, or of a differentiation of their roles temporally and/or spatially. A recent study on peroxidasins and peroxidasin-like proteins showed that human vascular peroxidasins are expressed in heart, and are putatively down-regulated by peroxidasin-like proteins (Peterfi et al., 2014). The peroxidasin-like protein consists of a peroxidase domain lacking the crucial enzyme activity and cell adhesion domains, such as leucine-rich repeats (LRRs) and immunoglobulin (Ig) domains. Hence, it is suggested that peroxidasin and peroxidasin-like protein can form complexes to inhibit the peroxidase activity of peroxidasin for proper regulation (Peterfi et al., 2014). The relationship between pxn-1 and $p x n-2$ in $C$. elegans may be similar to this interaction between peroxidasin and peroxidasin-like protein in the human heart. Further investigation of the relationship between pxn-1 and pxn-2 in neuronal development and maintenance and in attachments between tissues should prove rewarding.

\section{ACKNOWLEDGMENTS}

We thank Caenorhabditis Genetics Center (CGC) at University of Minnesota, USA for worm strains and Andrew Fire for the fire vectors. This research was supported by the National Research Foundation of Korea (NRF) grant 2010-0012373.

\section{REFERENCES}

Bhave, G., Cummings, C.F., Vanacore, R.M., Kumagai-Cresse, C., Ero-Tolliver, I.A., Rafi, M., Kang, J.S., Pedchenko, V., Fessler, L.I., Fessler, J.H., et al. (2012). Peroxidasin forms sulfilimine chemical bonds using hypohalous acids in tissue genesis. Nat. Chem. Biol. 8, 784-790.

Brenner, S. (1974). The genetics of Caenorhabditis elegans. Genetics $77,71-94$.
Dickson, B.J. (2002). Molecular mechanisms of axon guidance. Science 298, 1959-1964.

Fidler, A.L., Vanacore, R.M., Chetyrkin, S.V., Pedchenko, V.K., Bhave, G., Yin, V.P., Stothers, C.L., Rose, K.L., McDonald, W.H., Clark, T.A., et al. (2014). A unique covalent bond in basement membrane is a primordial innovation for tissue evolution. Proc. Natl. Acad. Sci. USA 111, 331-336.

Gotenstein, J.R., Swale, R.E., Fukuda, T., Wu, Z., Giurumescu, C.A., Goncharov, A., Jin, Y., and Chisholm, A.D. (2010). The C. elegans peroxidasin PXN-2 is essential for embryonic morphogenesis and inhibits adult axon regeneration. Development 137, 3603-3613.

Gupta, M.C., Graham, P.L., and Kramer, J.M. (1997). Characterization of alpha1(IV) collagen mutations in Caenorhabditis elegans and the effects of alpha1 and alpha2(IV) mutations on type IV collagen distribution. J. Cell Biol. 137, 1185-1196.

Jin, Y., Jorgensen, E., Hartwieg, E., and Horvitz, H.R. (1999). The Caenorhabditis elegans gene unc-25 encodes glutamic acid decarboxylase and is required for synaptic transmission but not synaptic development. J. Neurosci. 19, 539-548.

Knobel, K.M., Jorgensen, E.M., and Bastiani, M.J. (1999). Growth cones stall and collapse during axon outgrowth in Caenorhabditis elegans. Development 126, 4489-4498.

Mello, C., and Fire, A. (1995). DNA transformation. Methods Cell Biol. $48,451-482$

Mueller, B.K. (1999). Growth cone guidance: first steps towards a deeper understanding. Annu. Rev. Neurosci. 22, 351-388.

Nelson, R.E., Fessler, L.I., Takagi, Y., Blumberg, B., Keene, D.R., Olson, P.F., Parker, C.G., and Fessler, J.H. (1994). Peroxidasin: a novel enzyme-matrix protein of Drosophila development. EMBO J. 13, 3438-3447.

Peterfi, Z., and Geiszt, M. (2014). Peroxidasins: novel players in tissue genesis. Trends Biochem. Sci. 39, 305-307.

Peterfi, Z., Toth, Z.E., Kovacs, H.A., Lazar, E., Sum, A., Donko, A., Sirokmany, G., Shah, A.M., and Geiszt, M. (2014). Peroxidasinlike protein: a novel peroxidase homologue in the human heart. Cardiovasc. Res. 101, 393-399.

Peters, K., McDowall, J., and Rose, A.M. (1991). Mutations in the bli-4 (I) locus of Caenorhabditis elegans disrupt both adult cuticle and early larval development. Genetics 129, 95-102.

Poinat, P., De Arcangelis, A., Sookhareea, S., Zhu, X., Hedgecock, E.M., Labouesse, M., and Georges-Labouesse, E. (2002). A 
conserved interaction between beta1 integrin/PAT-3 and Nckinteracting kinase/MIG-15 that mediates commissural axon navigation in C. elegans. Curr. Biol. 12, 622-631.

Soudi, M., Zamocky, M., Jakopitsch, C., Furtmuller, P.G., and Obinger, C. (2012). Molecular evolution, structure, and function of peroxidasins. Chem. Biodivers 9, 1776-1793.

Tessier-Lavigne, M., and Goodman, C.S. (1996). The molecular biology of axon guidance. Science 274, 1123-1133.

Thacker, C., Peters, K., Srayko, M., and Rose, A.M. (1995). The bli4 locus of Caenorhabditis elegans encodes structurally distinct kex2/subtilisin-like endoproteases essential for early develop- ment and adult morphology. Genes Dev. 9, 956-971.

Thacker, C., Srayko, M., and Rose, A.M. (2000). Mutational analysis of bli-4/kpc-4 reveals critical residues required for proprotein convertase function in C. elegans. Gene 252, 15-25.

Topf, U., and Chiquet-Ehrismann, R. (2011). Genetic interaction between Caenorhabditis elegans teneurin ten-1 and prolyl 4 hydroxylase phy-1 and their function in collagen IV-mediated basement membrane integrity during late elongation of the embryo. Mol. Biol. Cell 22, 3331-3343.

Weiss, S.J. (2012). Peroxidasin: tying the collagen-sulfilimine knot Nat. Chem. Biol. 8, 740-741. 